\title{
Assessing the Quality of Patient Information for Cholesteatoma on the Video Sharing Platform YouTube
}

\author{
Rithvik Reddy ${ }^{1}$, Horace Cheng ${ }^{1}$, Nicholas Jufas ${ }^{1}$, and Nirmal Patel ${ }^{1}$ \\ ${ }^{1}$ University of Sydney
}

May 30, 2021

\begin{abstract}
Objectives: The objective of this study is to assess quality of the most popular cholesteatoma videos on YouTube using recognized scoring systems and to determine if video quality metrics correlated with video popularity based on likes and views Design: Cross sectional survey of available data Setting: Metadata acquisition using YouTube searches using Australian IP addresses Participants: Three independent neuro-otologists partaking in scoring videos Main outcome measures: Each video was viewed and scored by three independent assessors using both a novel tool to score the usefulness of the video as well as the validated DISCERN scoring tool. Popularity metrics were analyzed and compared to video popularity. Results: A total of 90 YouTube videos were analyzed with an average 55,292 views per video with an average of 271 likes and 22 dislikes. The inter-rater correlation was moderate with Fleiss-kappa score $0.42[\mathrm{P}<0.01]$ using a novel scoring tool for cholesteatoma and inter-rater correlation coefficient was $0.78[95 \% \mathrm{CI}=0.58-0.90]$ indicating good reliability for DISCERN scores. The overall video quality was poor with higher DISCERN scores found in videos uploaded from Academic Institutions. Conclusions: Informative video quality on YouTube on cholesteatoma is overall of poor quality. Videos with unclassified sources or more dislikes correlated poorly with video quality. Given the increase in patients turning to the internet for information regarding their health conditions, otology and otolaryngology societies should be encouraged to publish high quality YouTube videos on cholesteatoma and other ear conditions.
\end{abstract}

\section{Assessing the Quality of Patient Information for Cholesteatoma on the Video Sharing Platform YouTube}

Keywords:

Cholesteatoma, Patient Education, YouTube, Otology, Consent, Health Information

\section{Abstract:}

Objectives:

The objective of this study is to assess quality of the most popular cholesteatoma videos on YouTube using recognized scoring systems and to determine if video quality metrics correlated with video popularity based on likes and views

Design:

Cross sectional survey of available data

Setting:

Metadata acquisition using YouTube searches using Australian IP addresses

Participants: 
Three independent neuro-otologists partaking in scoring videos

Main outcome measures:

Each video was viewed and scored by three independent assessors using both a novel tool to score the usefulness of the video as well as the validated DISCERN scoring tool. Popularity metrics were analyzed and compared to video popularity.

Results:

A total of 90 YouTube videos were analyzed with an average 55,292 views per video with an average of 271 likes and 22 dislikes. The inter-rater correlation was moderate with Fleiss-kappa score $0.42[\mathrm{P}<0.01]$ using a novel scoring tool for cholesteatoma and inter-rater correlation coefficient was 0.78 [95\% CI $=0.58-0.90]$ indicating good reliability for DISCERN scores. The overall video quality was poor with higher DISCERN scores found in videos uploaded from Academic Institutions.

\section{Conclusions:}

Informative video quality on YouTube on cholesteatoma is overall of poor quality. Videos with unclassified sources or more dislikes correlated poorly with video quality. Given the increase in patients turning to the internet for information regarding their health conditions, otology and otolaryngology societies should be encouraged to publish high quality YouTube videos on cholesteatoma and other ear conditions.

Keywords: Cholesteatoma, Patient Education, YouTube, Otology, Consent, Health Information

Key points:

- Patients are increasingly using the internet and YouTube to gain access to educational material about their medical conditions

- The aim of this study is to evaluate the educational quality of YouTube content in regards to cholesteatoma

- The DISCERN tool and a novel video scoring tool to measure were used to evaluate 90 YouTube videos found using search terms concerning cholesteatoma

- The overall informative quality of the YouTube videos about cholesteatoma found was poor

- Otology and Otolaryngology societies should be encouraged to publish high quality, evidence-based patient education resources on platforms like YouTube

\section{Introduction}

The internet has changed the way in which patients access health information. More than half of all internet users are accessing their own health information online. Higher percentages of patients with chronic health conditions are turning to the internet for answers about their health $(1,2)$. Some studies have found that in over $80 \%$ of cases $(3,4)$, this information influences patient decisions regarding their chosen treatment.

YouTube is a video sharing platform with over two billion logged-in users (Alphabet Inc., Mountain View, California, USA) each month and every day people watch over a billion hours of videos and generate billions of views (5). YouTube allows users to upload, curate and comment on their own and others' content. The site has some content restriction oversight by the company but in general, videos are not validated or peer reviewed. Whilst the website facilitates dissemination of evidence-based healthcare information, it can also be a medium for biased, anecdotal and unregulated material from some users.

Anecdotal evidence of unproven alternative therapies and biased negative reporting of scientifically validated therapies is available to patients, and if they base their decision making solely on this information, there may be significant safety consequences (4). There are surveys showing that some patients trust internet sources of information more than their own physicians (6). 
Cholesteatoma is a chronic ear condition frequently requiring surgical management and post-operative surveillance (11). Patient participation in clinical decisions improves satisfaction (12) however, adherence to instructions requires an understanding of the condition and management options (13). The aim of this study is to analyze the quality of health information presented for patients with cholesteatoma on YouTube.

\section{Materials and Methods}

\section{Ethical Considerations}

Ethics approval was not required by the local Research Ethics Board as the information was global data already available in the public domain. All gathered data was de-identified for the analysis and publication. Researchers conformed to YouTube user guidelines of 2020.

\section{Trial design and setting}

Google Trends was searched in April 2020 for Cholesteatoma and related pathological diagnosis terms. Using these data, YouTube was then searched in May 2020 for "Cholesteatoma", "Ear Cholesteatoma", "Cholesteatoma Removal", "Cholesteatoma Surgery" and "Cholesteatoma Surgery Recovery". The searches were undertaken by three different authors using Google Chrome Version 83.0.4103.61. The cache of each computer, search history and cookies of the browser were reset before each search and the authors were not signed into their Google accounts. The default reference filter was chosen as most users do not adjust filters; a Virtual Private Network (VPN) was used with IP addresses across Australia.

The search was limited to the top six English language videos for each search term as determined by YouTube's relevance algorithm. The website results were displayed according to the default algorithm. To reproduce user search strategies, as clips were viewed and the site offered additional "up next" video suggestions, the top three suggested videos were also scored. The initial search, subsequent screening, analysis, and scoring of videos was performed independently by the three authors (NP and NJ both otologists, and $\mathrm{HC}$ otology Fellow).

\section{Metadata collection:}

YouTube provides additional information on published videos as popularity metrics. This 'metadata' was gathered during video scoring. The metrics included in this analysis were number of video views, cumulative user-based votes of approval or disapproval (likes or dislikes) that are assigned to videos, duration of the video and date the video was uploaded.

\section{Scoring criteria:}

The DISCERN instrument was used to evaluate the videos. The DISCERN tool was developed in 1999 in order to systematically judge the quality of patient information literature used to explain treatment options (7). The tool consists of 16 questions about both the reliability of the publication as well as the quality of information. Each question was rated from 1 to 5 with higher score representing more comprehensive explanation. The total score ranged from 16 to 80. Although the DISCERN tool was originally designed for written material, it has been used in the assessment of YouTube videos in medicine and also in otolaryngology $(8-10)$.

In addition to this, an Essential and Ideal Video Completeness (EIVC) Criteria was developed by the senior authors (NP and NJ) to be the minimum information points required by a patient to make an informed decision regarding consent for cholesteatoma treatment (Table 1). The three senior authors (NP, NJ and HC) have a combined experience of over 20 years in otologic practice in managing cholesteatoma. Audiovisual quality was assessed as either adequate or inadequate by the reviewers.

All videos were independently scored by the three senior authors (NP, NJ and HC). The scores were then collated by the fourth author (RR) to perform the statistical analysis. 


\section{Video classification:}

Videos were grouped based on the broad category of source: academic, private physician, patient, company/advertisement and unclassified. Videos were grouped based on the broad category of content: patient experience, patient-focused education, health care practitioner education, intra-operative videos and advertisements.

\section{Statistical methods}

Interrater reliability was determined for EIVC criteria and the DISCERN tool. The EIVC criteria was classed as an ordinal variable and the Fleiss-kappa statistical analysis was performed to determine the level of interobserver reliability. The DISCERN tool was classed as a continuous variable and an interclass correlation co-efficient was calculated. The DISCERN score and essential video scoring criteria were then compared with the video metrics, source and type using a one-way Welch ANOVA test, Pearson's product-moment correlation and Spearman's rank-order correlation.

The Statistical analysis was done with the Statistical Package for Social Sciences (SPSS) 25 software package (SPSS Inc., Chicago, IL).

Results

\section{Characteristics of included videos:}

After search and application of inclusion and exclusion criteria, a total of 90 YouTube videos were included for analysis. The characteristics of the videos are summarized in Table 2. The most common source was from an academic institution $(37.8 \%)$ followed by private physicians $(35.6 \%)$ and patient uploaded content (21.1\%). The most common content type was patient education (31.1\%), followed by intraoperative videos $(28.9 \%)$, patient experience videos $(23.3 \%)$ and finally health care practitioner videos $(16.7 \%)$. Of the 90 videos that were found, 18 videos were reviewed by all three reviewers and these videos were used to assess the inter-rater reliability and Fleiss-Kappa statistic.

Video quality assessment and inter-rater reliability:

In terms of Essential and Ideal Video Completeness Criteria, the mean score was 1.62 points out of a possible total 8 points, indicating poor video quality. In terms of inter-rater reliability of this metric across 3 raters, the inter-rater correlation was moderate with Fleiss-kappa score $0.42[\mathrm{P}<0.01]$

The average total DISCERN score was 31.3 (out of a possible 80), indicating a low reliability of the information presented. In the breakdown of the DISCERN score (see table 3), the mean scores for the categories representing stating explicit aims, achieving those aims, remaining relevant to the topic, having explicit sources of information and stating the date of the information performed well (scores above 2.5 points) with the majority of the remaining categories scoring less than 2 points. The inter-rater correlation coefficient was $0.78[95 \% \mathrm{CI}=0.58-0.90]$ indicating good reliability between raters.

Correlation of the DISCERN score and video metrics, video source and video type:

For the 90 YouTube videos found, the mean views were 55292.4, mean likes 270.7, mean dislikes 21.5 and mean duration of video 11.9 minutes.

A Pearson's product-moment correlation was run to assess the relationship between the total DISCERN score and video metrics (views, likes, dislikes and video length in minutes). A higher number of dislikes was correlated with a lower DISCERN score, $\mathrm{r}(98)=-0.24[\mathrm{p}=0.02]$. There was no statistically significant correlation found between the DISCERN score and the video views, likes or length.

A one-way Welch ANOVA was conducted to determine if DISCERN total score was different for groups with different video types or video sources (see Table 4). Mean DISCERN scores were not statistically significantly different between classified different video sources, however, Games-Howell post hoc analysis revealed that the mean increase in DISCERN scores from unclassified to academic sources $(15.85,95 \%$ CI [3.36, 28.34]) was 
statistically significant $(\mathrm{p}=-.006)$. A one-way Welch ANOVA showed there was no significant differences between the mean DISCERN scores amongst the different video types.

Correlation of the essential criteria scores and video metrics, sources and types:

A Spearman's rank-order correlation was run to assess the relationship between the EIVC criteria score and video metrics (views, likes, dislikes and video length in minutes). A higher number of dislikes was correlated with a lower essential criteria score, $\mathrm{r}_{\mathrm{s}}(98)=-0.217, \mathrm{p}=0.04$. There was no correlation with the video views, likes or video length with the EIVC scores.

A one-way Welch ANOVA was conducted to determine if the EIVC scores were different amongst the video source and video type groups. There was no statistically significant differences between the EIVC scores amongst the groups of video sources however, it was found that patient education type videos were scored significantly higher than intra-operative type videos $(\mathrm{p}=0.003)$.

\section{Discussion}

To our knowledge, this is the first in-depth study objectively assessing the quality and reliability of video information pertaining to cholesteatoma on YouTube. There were on average 55,292 views per video included in this study with videos having an average of 271 likes and only 22 dislikes.

In otolaryngology, YouTube content has been investigated for the educational value of videos about pediatric tonsillectomy, middle ear ventilation tubes, rhinoplasty and thyroid cancer $(8,14-16)$. Sorenson (16) found there was a low educational quality for YouTube videos describing pediatric adenotonsillectomy with even lower quality scores in videos from patient uploaded testimonial-type content. These findings were mirrored by Strychowsky et al (15), with study findings revealing physician-uploaded content being of a better educational quality than patient experience, surgical technique and news media videos. The findings of the current study confirm that videos uploaded by patients are of a lower educational value when compared to videos from academic sources.

The DISCERN tool was developed in 1999 in order to critically appraise the reliability and validity of published health information (7) and the DISCERN instrument has since been used generically in assessing YouTube sources in several studies $(8,9,14)$.

In addition to the DISCERN tool, the senior authors developed a disease specific Essential and Ideal Video Completeness Criteria (EIVC criteria) to represent the necessary video contents required in order to inform patients of all the elements of cholesteatoma and its management. Novel scoring systems have previously been developed to address the intricacies of disease factors being assessed such as Strychowsky et al who developed a usefulness checklist for validity of YouTube health information videos regarding pediatric tonsillectomy(15).

The overall educational quality of the videos examined was poor using both the DISCERN and EIVC Criteria. The DISCERN scores in this study showed a good reliability between raters and there was moderate correlation between raters in the EIVC criteria. The results show that a video's view count, length and like tally did not correlate with the DISCERN or EIVC criteria however, the more dislikes a video had, the lower the DISCERN or EIVC score.

In a systematic review addressing the health care information on YouTube, Madathil (4) et al found that although YouTube had the potential to be a great source of patient education, it was potentially also at significant risk of bias and misinformation. It was been recommended that professional organizations and government agencies (17) take more of an active role in uploading approved videos which are more likely to meet the criteria and guidelines required. Given the poor educational content found in this study, as well as other past otolaryngology studies $(8,15,16)$, the authors advocate for otolaryngology and otology societies working with patients in order to upload high quality patient education videos onto YouTube which surgeons can direct patients to view for more accurate information.

With regards to the limitations of this study, firstly it was limited to the English Language and only the top 6 search results were examined. The authors felt that this would generally match what the average 
patient would search for, but of course, many may search for more information. The geographical location of the YouTube user also affects the search findings and as such, our study may not be representative of the quality of videos found in other regions. The DISCERN instrument was originally created to assess written material and as such, has not been properly validated for use in the video educational material. Furthermore, the essential and ideal video completeness criteria is an unvalidated instrument based on replicating similar models of disease assessment used to analyze video educational content.

\section{Conclusion}

YouTube offers the opportunity of free and easy to access form of health information for patients but also the potential for biased, unvalidated misinformation potentially affecting patient decision making. At this time, the educational quality of YouTube videos pertaining to cholesteatoma is poor and content containing useful, evidence-based patient advice about cholesteatoma is lacking. The authors advocate that surgeons and surgical societies should upload evidence-based, unbiased and educational YouTube content about common surgical presentations and treatments to allow patients to make a more informed decision about their treatment.

\section{References}

1. Madrigal L, Escoffery C. Electronic Health Behaviors Among US Adults With Chronic Disease: CrossSectional Survey. Journal of medical Internet research. 2019;21(3):e11240-e.

2. Atkinson NL, Saperstein SL, Pleis J. Using the internet for health-related activities: findings from a national probability sample. J Med Internet Res. 2009;11(1):e4.

3. Fox S. The online health care revolution: How the web helps Americans Take better care of themselves. A Pew Internet and American Life Project Online Report. http://www pewinternet org/reports/pdfs/PIP_Health_Report pdf. 2000.

4. Madathil KC, Rivera-Rodriguez AJ, Greenstein JS, Gramopadhye AK. Healthcare information on YouTube: A systematic review. Health Informatics Journal. 2015;21(3):173-94.

5. Aslam S. YouTube by the Numbers (2020): Stats, Demographics \& Fun Facts: OmniCore; Feb 10, 2020 [Available from: https://www.omnicoreagency.com/youtube-statistics/.

6. Diaz JA, Griffith RA, Ng JJ, Reinert SE, Friedmann PD, Moulton AW. Patients' use of the Internet for medical information. Journal of general internal medicine. 2002;17(3):180-5.

7. Charnock D, Shepperd S, Needham G, Gann R. DISCERN: an instrument for judging the quality of written consumer health information on treatment choices. J Epidemiol Community Health. 1999;53(2):105-11.

8. Oremule B, Patel A, Orekoya O, Advani R, Bondin D. Quality and Reliability of YouTube Videos as a Source of Patient Information on Rhinoplasty. JAMA Otolaryngology-Head \& Neck Surgery. 2019;145(3):282-3.

9. Gokcen HB, Gumussuyu G. A Quality Analysis of Disc Herniation Videos on YouTube. World Neurosurg. 2019 .

10. Li HO-Y, Bailey A, Huynh D, Chan J. YouTube as a source of information on COVID-19: a pandemic of misinformation? BMJ Global Health. 2020;5(5):e002604.

11. Samadhiya M, Agarwal H, Vaidya S, Sharma JK. Outcome of Canal Wall Down Mastoidectomy: A Retrospective Review. Indian Journal of Otolaryngology and Head \& Neck Surgery. 2020.

12. Chewning B, Bylund CL, Shah B, Arora NK, Gueguen JA, Makoul G. Patient preferences for shared decisions: A systematic review. Patient Education and Counseling. 2012;86(1):9-18.

13. Kripalani S, Yao X, Haynes RB. Interventions to Enhance Medication Adherence in Chronic Medical Conditions: A Systematic Review. Archives of Internal Medicine. 2007;167(6):540-9. 
14. Aydin MA, Akyol H. Quality of Information Available on YouTube Videos Pertaining to Thyroid Cancer. Journal of Cancer Education. 2020;35(3):599-605.

15. Strychowsky JE, Nayan S, Farrokhyar F, MacLean J. YouTube: A good source of information on pediatric tonsillectomy? International journal of pediatric otorhinolaryngology. 2013;77(6):972-5.

16. Sorensen JA, Pusz MD, Brietzke SE. YouTube as an information source for pediatric adenotonsillectomy and ear tube surgery. International journal of pediatric otorhinolaryngology. 2014;78(1):65-70.

17. Singh AG, Singh S, Singh PP. YouTube for information on rheumatoid arthritis-a wakeup call? J Rheumatol. 2012;39(5):899-903.

\section{Table 1: Essential and Ideal Video Completeness Criteria for Cholesteatoma}

- Explanation of Cholesteatoma - definition with classification, etiology, epidemiology, clinical features/natural history, diagnosis, prognosis

- Treatment options - Observe if elderly or infirm or Surgery (see specific below)

- Pre-operative investigations - audiometry, Computed Topography +/- Magnetic Resonance Imaging

- Risks of operation - Facial Nerve Damage, Dizziness/Balance/Vertigo, Tinnitus, Taste disturbance, Incomplete eradication/recurrence, Hearing loss (Conductive or Sensorineural), Tympanic Membrane Perforation/Graft Failure, Pain, Infection, Bleeding

- Surgical goals and strategies for the operation

- Operation - surgical approaches (open/endoscopic) and canal wall-up/down

- Post-operative management and options

- Outcomes/expectations - healing, hearing, follow-up time

Table 2: Summary of included YouTube Videos relating to cholesteatoma search terms

\begin{tabular}{ll}
\hline Parameter & Total $(\mathbf{n = 9 0 )}$ \\
\hline Source Type & \\
Academic institution & $34(37.8 \%)$ \\
Private Physician & $32(35.6 \%)$ \\
Patient & $19(21.1 \%)$ \\
Unclassified & $3(3.3 \%)$ \\
Company & $2(2.2 \%)$ \\
Content Type & \\
Patient Education & $28(31.1 \%)$ \\
Intraoperative video & $26(28.9 \%)$ \\
Patient Experience & $21(23.3 \%)$ \\
Health care practitioner & $15(16.7 \%)$ \\
Viewer statistics & \\
Mean Views & 55292.4 \\
Median Views & 15386.5 \\
Mean Likes & 270.7 \\
Median Likes & 92 \\
Mean Dislikes & 21.5 \\
Median Dislikes & 5 \\
Mean duration (minutes) & 11.9 \\
Median duration (minutes) & 6.2 \\
\hline
\end{tabular}

Table 3: Mean scores of DISCERN questions 


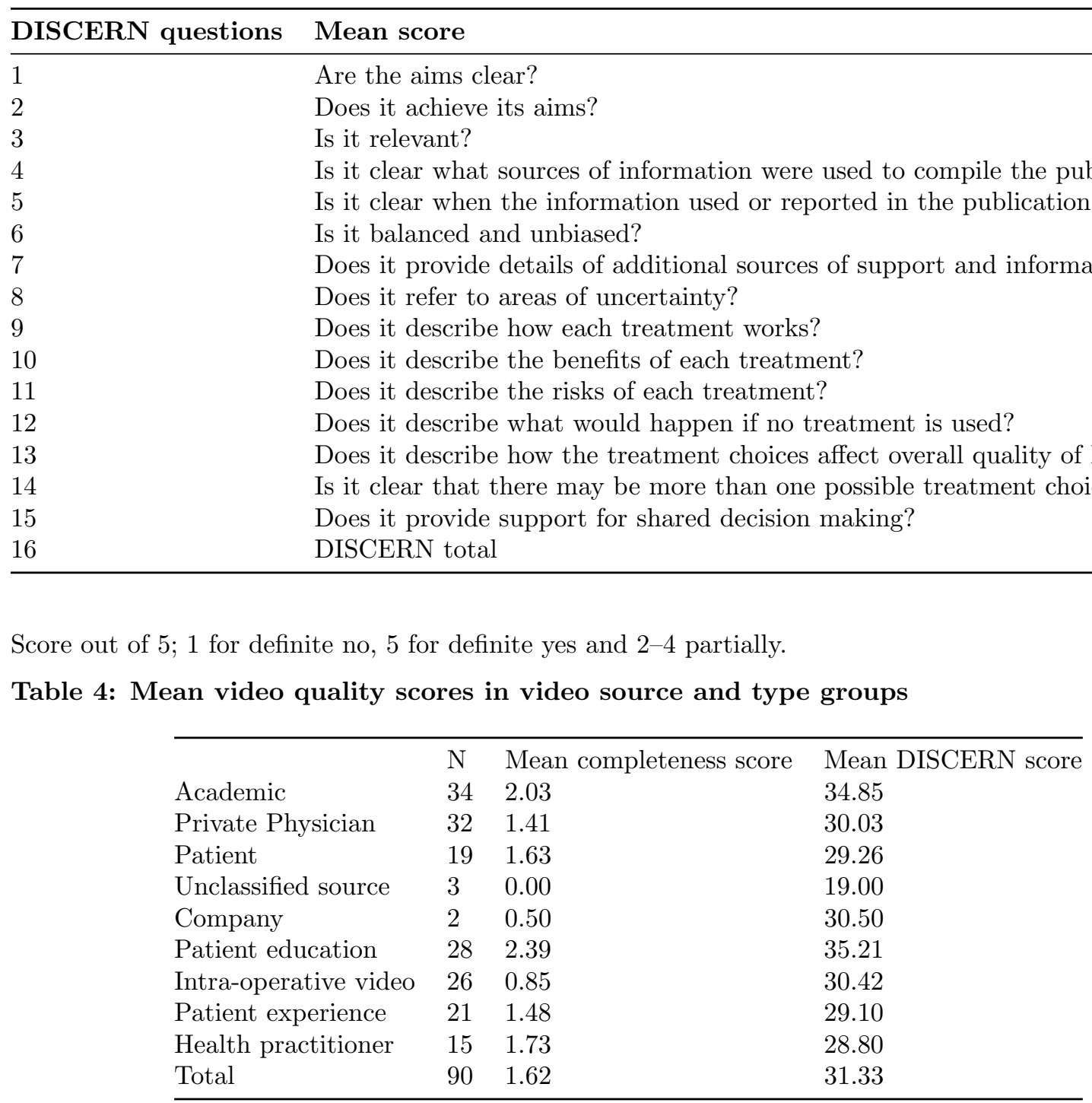

Word count total: 2425

Abstract: 243

Manuscript: 2182 